\title{
Susceptibility of Incisional Site Staphylococcus spp. to Three Common Perioperative Antimicrobials in Dogs Undergoing Clean Orthopaedic Procedures
}

\author{
Po-Yen Chou ${ }^{1}$ Duane Robinson ${ }^{1, *}$ Amy S. Kapatkin ${ }^{1}$ Steven E. Epstein ${ }^{1}$ Michelle A. Giuffrida ${ }^{1}$ \\ Barbara A. Byrne ${ }^{2}$ \\ ${ }^{1}$ Department of Surgical and Radiological Science, School of \\ Veterinary Medicine, University of California, Davis, California, \\ United States \\ 2 Department of Pathology, Microbiology and Immunology, School of \\ Veterinary Medicine, University of California, Davis, California, \\ United States

\begin{abstract}
Address for correspondence Po-Yen Chou, BVM, MS, DACVS-SA, Department of Surgical and Radiological Science, School of Veterinary Medicine, University of California-Davis, Davis, CA 95616-5270,
\end{abstract} \\ United States (e-mail: pchou@ucdavis.edu).
}

VCOT Open 2020;3:e158-e163.

\begin{abstract}
Keywords

- methicillin susceptibility

- Staphylococcus

- dogs

- perioperative antimicrobial therapy

Objectives The aim of this study was to evaluate the susceptibility pattern of the incisional site Staphylococcus spp. to three commonly used perioperative antimicrobials in dogs.

Materials and Methods A cohort of client-owned dogs was included in this prospective observational study. After induction of general anaesthesia and hair clipping, culture samples were collected from the incisional site of healthy dogs before orthopaedic surgery. The isolated colonies were identified. The susceptibility pattern of staphylococcal isolates was determined using Clinical and Laboratory Standard Institute standards. The staphylococcal susceptibilities to amoxicillin/clavulanic acid and cefpodoxime were compared with cefazolin using two-sided equality of proportions test.

Results A total of 391 bacterial isolates were identified in 115 dogs. Bacillus spp. ( $n=200)$ and Staphylococcus spp. $(n=95)$ were the most common bacteria isolated. Coagulase- positive and -negative staphylococci were isolated in 44 and 38\% of dogs respectively. Cefazolin, amoxicillin/clavulanic acid and cefpodoxime staphylococcal susceptibilities were 68,77 and $76 \%$ respectively. There were no significant differences in Staphylococcus spp. susceptibility between amoxicillin/clavulanic acid or cefpodoxime and cefazolin $(p=0.19, p=0.25)$. Thirty-six per cent of coagulase-negative staphylococci and $6 \%$ of coagulase-positive staphylococci isolated were methicillin resistant.

Conclusion Cefazolin, amoxicillin/clavulanic acid and cefpodoxime had similar susceptibilities for staphylococcal isolates. Routine use of amoxicillin/clavulanic acid or cefpodoxime in replace of cefazolin should be practiced with caution.
\end{abstract}

\footnotetext{
Current address: BluePearl Veterinary Partners, Seattle, Washington, United States.
}

received

May 12, 2020

accepted after revision

August 19, 2020
DOI https://doi.org/

10.1055/s-0040-1716847.

ISSN 2625-2325. (c) 2020 Georg Thieme Verlag KG

Stuttgart · New York
License terms

(c) (i) 


\section{Introduction}

Cefazolin (CFZ) is the most commonly used perioperative antibiotic medication in human and veterinary orthopaedic surgery. It has low toxicity, high bone and joint tissue concentrations, and its use is associated with decreased rates of surgical site infections (SSI) compared with dogs that receive no perioperative antimicrobials. ${ }^{1-3}$ Second- and third-generation cephalosporins or potentiated penicillin are also used as perioperative antimicrobials. ${ }^{4,5}$ They may have the advantage over CFZ because they tend to be effective against $\beta$-lactamase producing Staphylococcus, ${ }^{6}$ whose prevalence is increasing. ${ }^{7,8}$ However, information about their effectiveness as perioperative prophylactic antibiotic medications for orthopaedic procedures in dogs is lacking.

Susceptibility patterns of potential bacteria at the surgical site are one of the considerations for selecting perioperative antimicrobials. ${ }^{9}$ To the author's knowledge, studies conducted for commensal staphylococci susceptibility patterns have specifically investigated methicillin-resistant staphylococci colonization, characterized by the presence of mec $\mathrm{A}$ gene or oxacillin resistance interpreted by the culture and sensitivity report. ${ }^{7,8}$ The susceptibility pattern of incisional site skin flora in dogs has not been evaluated in the literature. The purpose of the study was to evaluate the susceptibility of cutaneous staphylococci to commonly used prophylactic antimicrobials isolated from planned incision sites in dogs undergoing clean orthopaedic procedures. The null hypothesis tested was that staphylococcal isolates retrieved from planned incision sites would have no difference in susceptibility to cefpodoxime (CPD) and amoxicillin/clavulanic acid (AMC) than to CFZ.

\section{Materials and Methods}

This was a prospective observational study of healthy clientowned dogs. Dogs over a year of age that underwent clean orthopaedic surgery between November 2015 and May 2016 were eligible for inclusion. Dogs were excluded if they had received systemic or topical treatments for dermatological condition, systemic antimicrobial treatments within 4 weeks of presentation, had undergone a surgical procedure within 4 weeks of presentation, had a confirmed diagnosis of endocrine diseases or had skin lesions discovered after clipping. Signed informed owner consent was obtained. The study protocol was approved by the institutional animal care and use committee (protocol no 18309). Once enrolled, data collected included breed, sex, age, weight, urgency of surgery, classified as elective (surgeries for naturally occurring orthopaedic disease) or urgent (surgeries for fractures or luxations following a traumatic event), and anatomic site.

Dogs were anaesthetized, and the planned surgical incision site was clipped using a sterile no 40 clipper blade (Oster, Baca Raton, Florida, United States). The person preparing the site and taking the sample wore sterile gloves. Excess hair was vacuumed from the site without skin contact. A culture sample of the planned incision site was collected using a sterile contact agar plate (RODAC plate, ThermoFisher
Scientific, Waltham, Massachusetts, United States) containing $5 \%$ sheep blood. ${ }^{10}$ The entire surface of the agar plate was applied to the skin for 5 seconds. The plate was incubated in $5 \% \mathrm{CO}_{2}$ at $35^{\circ} \mathrm{C}$ for 24 hours, and the bacterial organisms were subcultured to obtain pure bacterial isolates. The pure bacterial isolates were used for identification and determination of antimicrobial susceptibility. Isolate identification utilized a combination of colony morphology characteristics, haemolytic reaction, Gram stain results, spot catalase testing and coagulase testing. Further characterization of isolate identity was based on results from conventional biochemical testing (API, BioMérieux, Durham, North Carolina, United States) (tubed media, catalase reaction, identification strips) or matrix-assisted time-of-flight mass spectrometry (Biotyper 3.0, Bruker, Fremont, California, United States). The susceptibilities of identified bacteria were determined using a broth microdilution technique (Sensititre Companion Animal Gram Positive COMPGP1G Vet AST plate, ThermoFisher Scientific, Waltham, Massachusetts, United States). Quality control testing was performed weekly using the following organisms: Staphylococcus aureus ATCC 29213; Enterococcus faecalis ATCC 29212, Escherichia coli ATCC 25922, Pseudomonas aeruginosa ATCC 27853, and E. coli ATCC 35218. Susceptibility, intermediate susceptibility and resistance of staphylococcal isolates were determined using standards established by the Clinical Laboratories Standards Institute for Animals. ${ }^{11}$ Staphylococcus intermedius and Staphylococcus pseudintermedius identified with matrix-assisted timeof-flight mass spectrometry were reported together as Staphylococcus intermedius group (SIG). No CPD breakpoint has been established for coagulase-negative (CoNS), so the breakpoint for S. pseudintermedius was used to help determine susceptibility. Oxacillin-resistant isolates were reported to be resistant to methicillin and all other $\beta$-lactam antimicrobials, regardless of individual $\beta$-lactam antimicrobial susceptibility testing result. ${ }^{11}$

Isolates interpreted to have intermediate susceptibility were considered resistant for statistical analysis. Descriptive statistics were calculated for all measured variables and reported as number (per cent), median (interquartile range) or mean (standard deviation); a combined test of skewness and kurtosis was used to evaluate normal distribution of continuous variables. Population rates of staphylococci susceptible to CFZ, CPD and AMC were estimated with surrounding exact binomial 95\% confidence intervals (CI). Two-sided equality of proportions test was used to test the null hypotheses that the rate of staphylococci susceptibility to CFZ did not differ significantly from the rate of susceptibility to CPD or from the rate of susceptibility to AMC. Antimicrobial susceptibilities of CFZ, CPD and AMC were compared across staphylococcal species using Fisher's exact tests. Logistic regression was performed to explore dog variables (age, body weight, sex, anatomic surgical site and urgency of surgery [classified as elective or emergent] ) associated with isolation of CFZ-resistant staphylococci. All tests were two-sided, with $p<0.05$ considered significant. Analyses were performed with computer software (Stata Statistical Software: Release 14, College Station, Texas, United States). 
e160 Susceptibility Pattern of the Incisional Site Staphylococcus spp. Chou et al.

\section{Results}

One-hundred-fifteen dogs that underwent clean orthopaedic surgery were enrolled in the study. The median age was 4 years (interquartile range:, 1-8). Commonly represented breeds included mixed breeds $(38 / 115,33 \%)$, Labrador Retriever (19/115, 16\%), Pitbull Terrier (6/115, 5\%), German Shepherd $\operatorname{dog}(6 / 115,5 \%)$ and Bulldog $(4 / 115,3 \%)$. Sex distribution was female spayed $(54 / 115,47 \%)$, male castrated $(43 / 115,37 \%)$, male intact $(10 / 115,9 \%)$ and female intact $(8 / 115,7 \%)$. Mean body weight was $25.9 \pm 14.3 \mathrm{~kg}$. The surgical orthopaedic disease were categorized as elective (77/115, 67\%), which included cranial cruciate ligament disease $(60 / 115,52 \%)$ medial patella luxation $(7 / 115,6 \%)$ hip dysplasia $(4 / 115,3 \%)$, elbow dysplasia $(6 / 115,5 \%)$ and urgent $(38 / 115,33 \%)$, which included traumatic fracture $(32 / 115,28 \%)$ and luxation $(6 / 115,5 \%)$. Surgical sites sampled were stifle $(68 / 115,59 \%)$, elbow joint $(17 / 115,15 \%)$, radius/ulna $(9 / 115,8 \%)$, tibia/fibula $(7 / 115,6 \%)$, humerus $(5 / 115,4 \%)$, femur $(5 / 115,4 \%)$ and coxofemoral joint $(4 / 115$, $3 \%$ ). The right limb was sampled in 59/115 (51\%), and the left limb was sampled in 56/115 (49\%) dogs.

Bacterial cultures resulted in a growth of 391 bacterial isolates in 115 dogs. One (1\%) dog had no growth. Eight (7\%) dogs grew one bacterial isolate, 27 (23\%) dogs grew two different isolates, 27 (23\%) dogs grew three different isolates, 27 (23\%) dogs grew four different isolates, 16 (14\%) dogs grew five different isolates and $9(8 \%)$ dogs grew six or more isolates.

Staphylococcus spp. were isolated from culture samples of 83/115 (72\%) dogs. Twelve dogs grew two different staphylococcal isolates, and the remaining 71 dogs grew a single staphylococcal isolate. Nine isolates identified as $S$. intermedius and $38 \mathrm{~S}$. pseudintermedius isolates were reported as SIG. The prevalence of bacterial isolates is shown in - Table $\mathbf{1}$.

On univariable logistic regression analysis, none of the following factors were significantly associated with growing a CFZ-resistant Staphylococcus spp.: age, sex, body weight, urgency of surgery or anatomic surgical site.

Among the 95 staphylococcal isolates, 65 (68\%, 95\% CI: 59.1-77.8) were susceptible to CFZ. In the 12 dogs that grew two different staphylococci, both isolates were susceptible in five dogs, both isolates were resistant in two dogs, and in the remaining five dogs, one isolate was susceptible, and the other was resistant. Among the 83 dogs from which Staphylococcus spp. were isolated, 28 (33.7\%, 95\% CI: 23.6-43.9) had at least one CFZ-resistant strain.

Among the 95 staphylococcal isolates, 73 (77\%, 95\% CI: 68.4-85.3) were susceptible to CPD. The difference in rate of staphylococcal susceptibility to CPD versus CFZ was $8.4 \%$ (95\% CI: 4.2-21.0), which was not significant $(p=0.19)$. Among the 83 dogs from which Staphylococcus spp. were isolated, 20 (24\%, 95\% CI: 15.5-33.9) dogs grew at least one CPD-resistant strain, which did not differ significantly $(p=0.17)$ from the proportion of dogs that grew at least one CFZ-resistant strain.

Among the 88 staphylococcal isolates for which AMC susceptibility results were available, 67 (76\%, 95\% CI:
Table 1 Distribution of 391 bacterial isolates isolated from the skin of 115 dogs underwent orthopaedic surgical procedures

\begin{tabular}{|l|l|l|}
\hline Isolate & $\begin{array}{l}\text { No. of } \\
\text { isolates }\end{array}$ & $\begin{array}{l}\text { No. (\%) of } \\
\text { dogs }\end{array}$ \\
\hline Bacillus spp. & 200 & $90(78)$ \\
\hline Staphylococcus spp. & 95 & $83(72)$ \\
\hline CoNS & 44 & $44(38)$ \\
\hline CoPS & 51 & $51(44)$ \\
\hline S. intermedius group & 47 & $47(41)$ \\
\hline S. aureus & 4 & $4(3)$ \\
\hline Micrococcus spp. & 34 & $26(23)$ \\
\hline Streptococcus spp. & 16 & $13(11)$ \\
\hline Non-Enterobacteriaceae spp. & 15 & $14(12)$ \\
\hline Enterococcus spp. & 11 & $11(10)$ \\
\hline Corynebacterium spp. & 7 & $7(6)$ \\
\hline Acinetobacter spp. & 6 & $6(5)$ \\
\hline Klebsiella oxytoca & 1 & $1(1)$ \\
\hline Pasteurella stomatis & 1 & $1(1)$ \\
\hline Escherichia coli & 1 & $1(1)$ \\
\hline Moraxella spp. & 1 & $1(1)$ \\
\hline Streptomyces spp. & 1 & $1(1)$ \\
\hline Ralstonia spp. & 1 & $1(1)$ \\
\hline Pantoea spp. & 1 & $1(1)$ \\
\hline Sile & & (1) \\
\hline
\end{tabular}

Abbreviations: CoNS, coagulase-negative staphylococci; CoPS, coagulase-positive staphylococci.

67.2-85.0) were susceptible to AMC. The difference in rate of staphylococcal susceptibility to AMC versus CFZ was $7.7 \%$ (95\% CI: 5.2-20.6), which was not significant $(p=0.25)$. Among the 76 dogs that had at least one Staphylococcus spp. isolated with AMC susceptibility reported, 19 (25\%, 95\% CI: 15.3-34.7) dogs grew at least one AMCresistant strain, which did not differ significantly $(p=0.23)$ from the proportion of dogs that grew at least one CFZ-resistant strain.

Among the 95 staphylococcal isolates, 36\% (16/44) of CoNS and 6\% (3/47) of SIG were resistant to oxacillin. No Staphylococcus aureus isolates were resistant to oxacillin. The oxacillin susceptibility differed significantly according to staphylococcal species; CoNS had a lower rate of oxacillin susceptibility compared with other strains $(p=0.001)$ (-Table 2).

\section{Discussion}

When evaluating all bacterial isolates, Staphylococcus spp. were the second most commonly isolated bacteria from planned incision sites. The isolated staphylococci demonstrated similar susceptibility patterns to CFZ, AMC and CFD. Therefore, the null hypothesis was accepted. In vitro testing showed that dermal staphylococci isolates retrieved from 
Table 2 Antimicrobial susceptibility for 95 Staphylococcus spp. isolated from the skin of 83 dogs underwent orthopaedic surgery. Amoxicillin/clavulanate susceptibility was not available for seven isolates

\begin{tabular}{|l|l|l|l|l|}
\hline & $\begin{array}{l}\text { CoNS } \\
\boldsymbol{n}=\mathbf{4 4}(\%)\end{array}$ & $\begin{array}{l}\text { SIG } \\
\boldsymbol{n}=\mathbf{4 7}(\%)\end{array}$ & $\begin{array}{l}\text { S. aureus } \\
\boldsymbol{n}=\mathbf{4}(\%)\end{array}$ & $p$-Value \\
\hline Cefazolin & $22 / 44(50)$ & $40 / 47(85)$ & $3 / 4(75)$ & 0.001 \\
\hline Cefpodoxime & $27 / 44(61)$ & $43 / 47(91)$ & $3 / 4(75)$ & 0.002 \\
\hline Amoxicillin/Clavulanic acid & $23 / 41(56)$ & $41 / 44(93)$ & $3 / 3(100)$ & $<0.001$ \\
\hline Oxacillin & $28 / 44(64)$ & $44 / 47(94)$ & $4 / 4(100)$ & 0.001 \\
\hline
\end{tabular}

Abbreviations: CoNS, coagulase-negative staphylococci; SIG, Staphylococcus intermedius group; S. aureus, Staphylococcus aureus. Note: $p$-Value $<0.05$ indicates significant difference in susceptibility across CoNS, SIG and S. aureus group.

planned incision sites had no difference in susceptibility to CPD and AMC than to CFZ.

Breed, surgical site, age, sex, body weight, urgency of surgery or anatomic site did not have a significant effect on the CFZ susceptibility of staphylococcal isolates. In a previous study, bulldogs were identified to have a significantly higher risk of having methicillin-resistant Staphylococcus pseudintermedius (MRSP) colonization and SSI. The differences in our results when compared with this previous study are likely due to different sample sizes and sampling methods. ${ }^{12}$ In the present study, only cases referred to one institution were included, and samples from the incision site were collected. Additionally, only dogs that received prior antimicrobial treatment within 4 weeks or with skin lesions were excluded. A more detailed screening process including information such as antimicrobial treatment status of other animals and people in the same household, and husbandry environment, may have helped capture a more accurate image on the relationship between MRSP colonization status and incisional site bacteria.

Bacillus spp. were the most common incisional site bacteria isolated. A high prevalence of Bacillus spp. at these sites has been reported in previous studies using culture-based ${ }^{13}$ and molecular biology methods using 16 s ribosomal ribonucleic acid amplification. ${ }^{14}$ Bacillus organisms are widely distributed in the environment and found in decaying organic matter, dust, vegetables and water. Bacillus spp. rarely causes orthopaedic SSIs. Except for Bacillus cereus and Bacillus anthracis, Bacillus spp. have low virulence and SSIs caused by Bacillus spp. were only reported in immunocompromised people, ${ }^{15}$ and SSIs caused by Bacillus spp. have not been reported in the veterinary literature.

The prevalence of coagulase-positive staphylococci (CoPS) and CoNS in this study was similar to those reported in previous veterinary studies. One study collected samples from multiple anatomic sites reported the isolation of CoPS in 37 of $50(74 \%)$ dogs with healthy skin. ${ }^{16}$ Other veterinary studies reported 33 to $100 \%$ isolation rates of CoNS in healthy dogs. ${ }^{13,16,17}$ In a human study, CoNS comprised $59.6 \%$ of total isolates from planned incision sites. ${ }^{18}$ Differences between published studies are likely due to the variation in the sampling method, and the anatomic sites sampled. One previous study showed that Staphylococcus spp. demonstrated anatomic niche preferences, and S. pseudintermedius is more often isolated from the perineum area, larynx and rectum. ${ }^{12,17}$ Samples from these other anatomic sites may have resulted in a higher isolation rate in the present study. However, the current study aimed to investigate the susceptibility of perioperative antimicrobial against bacteria present on incision sites; therefore, no samples were taken from other anatomic sites.

Six per cent of SIG isolated were oxacillin resistant. Due to the study design, a direct relationship between the MRSP colonization status and the bacteria present on the incision site could not be determined. In one previous study, the specificity of the incisional site sample for identifying MRSP carrier status was only $29.3 \%^{12}$; therefore, the actual number of dogs carrying methicillin-resistant SIG is likely to be higher. In addition, $36 \%$ of CoNS isolated in the present study were oxacillin resistant. This finding is similar to that in humans, where $28.6 \%$ of CoNS isolates were oxacillin resistant. ${ }^{18}$ Although traditionally regarded to be of low virulence, recognition of the role of CoNS as pathogens in veterinary dermatology is emerging, ${ }^{19}$ and the importance of CoNS in orthopaedic surgery should not be overlooked. Coagulase-negative staphylococci have been reported to be the causative bacteria in 12.7 to $14.2 \%$ of canine tibial plateau levelling osteotomy infections, ${ }^{20,21}$ in $22 \%$ of human fracture fixation infections, ${ }^{22}$ and up to $50 \%$ of human total hip arthroplasty infections. ${ }^{23,24}$ The high oxacillin resistance rate of CoNS observed in this study may provide one possible explanation for the high proportion of CoNS infections in human and veterinary orthopaedic surgery.

Staphylococcal isolates tested in the study showed similar susceptibilities to CPD, CFZ and AMC. The result is likely due to the high proportion of methicillin-resistant staphylococci. The addition of clavulanic acid to amoxicillin and the development of CPD helped increase the inhibition of $\beta$-lactamase and therefore have an improved coverage of methicillin susceptible staphylococci. ${ }^{22,25,26}$ However, several bacterial adaptation to $\beta$-lactamase have been discovered over the past decades, ${ }^{6}$ with the mec A gene coded altered penicillinbinding protein $2 \mathrm{a}$ being the most important one. The presence of penicillin-binding protein $2 a$ in staphylococci is responsible for the methicillin resistance phenotype and confers intrinsic resistance to all $\beta$-lactams. ${ }^{27}$

The prevalence of multidrug-resistant staphylococcal colonization and infection is increasing, ${ }^{28}$ but the susceptibility of incisional site Staphylococcus in dogs has not been reported to the authors' knowledge. The selection of 
antimicrobial for perioperative prophylactic administration should consider multiple factors, and the results of the current study should only be a reference. Degree of contamination, prior antimicrobial usage, targeted pathogenic bacteria, tissue concentration, institutional antimicrobial resistance pattern, cost and side effects should all taken into consideration. Pharmacokinetic studies to determine the optimal dosage and interval for prophylactic administration in dogs are lacking for AMC and CPD, but are available for CFZ. ${ }^{1,25}$ However, a direct comparison of the use of different medications in dogs is lacking. Human studies showed no difference in the SSI rate between prophylactic AMC, CPD and CFZ in human hip arthroplasty, knee arthroplasty and laparoscopic gynaecology surgery. ${ }^{29,30}$

Using the standard for interpreting cumulative antibiogram, ${ }^{31}$ the three antimicrobials evaluated would be classified as fair to good for in vitro efficacy against incisional site staphylococci. Although the antimicrobial susceptibility may seem low, the antimicrobials evaluated in the present study still have a high susceptibility against methicillin-susceptible staphylococci. The findings of this study may help to explain a previous study that shown the positive carrier status of MRSP being a risk factor for the development of SSI after tibial plateau levelling osteotomy. ${ }^{12}$ Best practices to prevent SSI in patients colonized with a resistant staphylococci remain unknown. Preoperative decontamination for human patients with methicillin-resistant Staphylococcus aureus colonization has been shown to be effective in preventing SSIs. ${ }^{32}$ Comparable information is unavailable in veterinary medicine and would require further investigation.

This study had several limitations. The confidence intervals around susceptibility rates and rate differences suggest that significant differences could yet exist between antimicrobials, although such modest differences would need to be correlated with SSI rates to determine their clinical significance. In this study, short- and long-term patient follow-up was not conducted, and the relationship between skin culture status and SSI was not examined. Multi-site research over a broader geographic area might provide additional information to guide clinical practice. Dogs in this study had no pathologic skin lesions around the incision site, and cultures were collected before the skin was surgically scrubbed. Bacteria seen on the culture may have included bacteria residing on the residual hair after clipping. Additionally, the isolation rate and susceptibility to CFZ, AMC and CFD after skin closure may have been different since perioperative antimicrobial prophylaxis is used in an effort to decrease total bacterial load throughout the procedure. Only conventional agar-based culture methods were used to detect bacteria. Recent advancements in molecular biology allowed bacterial identification using gene sequencing, ${ }^{33}$ which provides more detailed qualitative information to identify bacterial diversity. The currently used culture-based methods, although limited in breadth for the types of organisms detected and host-microbe interaction, have the advantage of quantifying absolute cell abundances of culturable microbes.

In conclusion, we found that $68 \%$ of staphylococci isolated from planned, clean, orthopaedic incision sites to be suscep- tible to CFZ. Cefpodoxime and AMC did not provide additional susceptibility coverage for isolated staphylococci. Based on the result of the present study, the lack of pharmacokinetic data, unknown added benefit for preventing SSI and antimicrobial stewardship, routine use of AMC or CPD in replace of CFZ should be practiced with caution.

\section{Authors' Contributions}

All authors contributed to the conception of the study, study design, data acquisition, analysis and interpretation. All authors drafted the revised and approved the submitted manuscript.

\section{Funding}

The study was supported by a grant from the Center for Companion Animal Health, School of Veterinary Medicine, University of California, Davis. The research was supported by the Center of Companion Animal Health, School of Veterinary Medicine, University of California, Davis.

\section{Conflict of Interest}

None declared.

\section{Acknowledgment}

The authors thank Dr Crystal Loranc, Mr Nathan Ortiz for technical assistance and Dr Denis Marcellin-Little for advice on manuscript preparation.

\section{References}

1 Marcellin-Little DJ, Papich MG, Richardson DC, DeYoung DJ. Pharmacokinetic model for cefazolin distribution during total hip arthroplasty in dogs. Am J Vet Res 1996;57(05):720-723

2 Verwilghen D, Singh A. Fighting surgical site infections in small animals: are we getting anywhere? Vet Clin North Am Small Anim Pract 2015;45(02):243-276, v

3 Whittem TL, Johnson AL, Smith CW, et al. Effect of perioperative prophylactic antimicrobial treatment in dogs undergoing elective orthopedic surgery. J Am Vet Med Assoc 1999;215(02): 212-216

4 Aiken MJ, Hughes TK, Abercromby RH, Holmes MA, Anderson AA. Prospective, randomized comparison of the effect of two antimicrobial regimes on surgical site infection rate in dogs undergoing orthopedic implant surgery. Vet Surg 2015;44(05):661-667

5 Pratesi A, Moores AP, Downes C, Grierson J, Maddox TW. Efficacy of postoperative antimicrobial use for clean orthopedic implant surgery in dogs: a prospective randomized study in 100 consecutive cases. Vet Surg 2015;44(05):653-660

6 Mealey KL. Penicillins and beta-lactamase inhibitor combinations. J Am Vet Med Assoc 2001;218(12):1893-1896

7 Priyantha R, Gaunt MC, Rubin JE. Antimicrobial susceptibility of Staphylococcus pseudintermedius colonizing healthy dogs in Saskatoon, Canada. Can Vet J 2016;57(01):65-69

8 Moodley A, Damborg P, Nielsen SS. Antimicrobial resistance in methicillin susceptible and methicillin resistant Staphylococcus pseudintermedius of canine origin: literature review from 1980 to 2013. Vet Microbiol 2014;171(3-4):337-341

9 Bratzler DW, Dellinger EP, Olsen KM, et al; American Society of Health-System Pharmacists (ASHP) Infectious Diseases Society of America (IDSA) Surgical Infection Society (SIS) Society for Healthcare Epidemiology of America (SHEA). Clinical practice guidelines for antimicrobial prophylaxis in surgery. Surg Infect (Larchmt) 2013;14(01):73-156 
10 Osuna DJ, DeYoung DJ, Walker RL. Comparison of three skin preparation techniques. Part 2: clinical trial in 100 dogs. Vet Surg 1990;19(01):20-23

11 Clinical and Laboratory Standards Institute Performance Standards for Antimicrobial Disk and Dilution Susceptibility Tests for Bacterial Isolates from Animals. 3rd Edition Wayne, PA: Clinical and Laboratory Standard Institute; 2015

12 Nazarali A, Singh A, Moens NMM, et al. Association between methicillin-resistant Staphylococcus pseudintermedius carriage and the development of surgical site infections following tibial plateau leveling osteotomy in dogs. J Am Vet Med Assoc 2015;247 (08):909-916

13 Ihrke PJ, Schwartzman RM, McGinley K, Horwitz LN, Marples RR. Microbiology of normal and seborrheic canine skin. Am J Vet Res 1978;39(09):1487-1489

14 Torres S, Clayton JB, Danzeisen JL, et al. Diverse bacterial communities exist on canine skin and are impacted by cohabitation and time. PeerJ 2017;5(10):e3075

15 Akesson A, Hedström SA, Ripa T. Bacillus cereus: a significant pathogen in postoperative and post-traumatic wounds on orthopaedic wards. Scand J Infect Dis 1991;23(01):71-77

16 Griffeth GC, Morris DO, Abraham JL, Shofer FS, Rankin SC. Screening for skin carriage of methicillin-resistant coagulase-positive staphylococci and Staphylococcus schleiferi in dogs with healthy and inflamed skin. Vet Dermatol 2008;19(03):142-149

17 Ma GC, Worthing KA, Ward MP, Norris JM. Commensal staphylococci including methicillin-resistant Staphylococcus aureus from dogs and cats in remote New South Wales, Australia. Microb Ecol 2020;79(01):164-174

18 Daeschlein G, Napp M, Layer F, et al. Antimicrobial efficacy of preoperative skin antisepsis and clonal relationship to postantiseptic skin-and-wound flora in patients undergoing clean orthopedic surgery. Eur J Clin Microbiol Infect Dis 2015;34(11): 2265-2273

19 Cain CL, Morris DO, Rankin SC. Clinical characterization of Staphylococcus schleiferi infections and identification of risk factors for acquisition of oxacillin-resistant strains in dogs: 225 cases (20032009). J Am Vet Med Assoc 2011;239(12):1566-1573

20 Savicky R, Beale B, Murtaugh R, Swiderski-Hazlett J, Unis M. Outcome following removal of TPLO implants with surgical site infection. Vet Comp Orthop Traumatol 2013;26(04):260-265

21 Gallagher AD, Mertens WD. Implant removal rate from infection after tibial plateau leveling osteotomy in dogs. Vet Surg 2012;41 (06):705-711
22 Neu HC, Fu KP. Clavulanic acid, a novel inhibitor of beta-lactamases. Antimicrob Agents Chemother 1978;14(05):650-655

23 Norton TD, Skeete F, Dubrovskaya Y, Phillips MS, Bosco JD III, Mehta SA. Orthopedic surgical site infections: analysis of causative bacteria and implications for antibiotic stewardship. Am J Orthop 2014;43(05):E89-E92

24 Lutro O, Langvatn $\mathrm{H}$, Dale $\mathrm{H}$, et al. Increasing resistance of coagulase-negative staphylococci in total hip arthroplasty infections: 278 THA-revisions due to infection reported to the Norwegian Arthroplasty Register from 1993 to 2007. Adv Orthop 2014; 2014(03):580359-7

25 Reading C, Hepburn P. The inhibition of staphylococcal betalactamase by clavulanic acid. Biochem J 1979;179(01):67-76

26 Frampton JE, Brogden RN, Langtry HD, Buckley MM. Cefpodoxime proxetil. A review of its antibacterial activity, pharmacokinetic properties and therapeutic potential. Drugs 1992;44(05):889-917

27 Fernandes R, Amador P, Prudêncio C. $\beta$-Lactams: chemical structure, mode of action and mechanisms of resistance. Rev Med Microbiol 2013;24(01):7-17

28 Morris DO, Loeffler A, Davis MF, Guardabassi L, Weese JS. Recommendations for approaches to methicillin-resistant staphylococcal infections of small animals: diagnosis, therapeutic considerations and preventative measures.: Clinical Consensus Guidelines of the World Association for Veterinary Dermatology. Vet Dermatol 2017; 28(03):304-e69

29 Mauerhan DR, Nelson CL, Smith DL, et al. Prophylaxis against infection in total joint arthroplasty. One day of cefuroxime compared with three days of cefazolin. J Bone Joint Surg Am 1994;76(01):39-45

30 Cormio G, Di Fazio F, Cacciapuoti C, Bettocchi S, Borraccino L, Selvaggi L. Prospective randomized study comparing amoxicillinclavulanic acid with cefazolin as antimicrobial prophylaxis in laparotomic gynecologic surgery. Acta Obstet Gynecol Scand 2003;82(12):1130-1134

31 Aucoin DP. Target; The Antimicrobial Guide to Effective Treatment. 3rd edition. Port Huron, MI: North American Comependiums Inc; 2007:iii-iv

32 Williams DM, Miller AO, Henry MW, Westrich GH, Ghomrawi HMK. Cost-effectiveness of Staphylococcus aureus decolonization strategies in high-risk total joint arthroplasty patients. J Arthroplasty 2017;32(9S):S91-S96

33 Rodrigues Hoffmann A, Patterson AP, Diesel A, et al. The skin microbiome in healthy and allergic dogs. PLoS One 2014;9(01): e83197 